American Journal of Applied Sciences 5 (6): 742-746, 2008

ISSN 1546-9239

(C) 2008 Science Publications

\title{
Lipid Profile of Gymnasts of the Jordan National Team
}

\author{
${ }^{1}$ Ahmad Bani Ata, ${ }^{2}$ Kamal Mansi, ${ }^{3}$ Talal Aburjai \\ ${ }^{1}$ Department of Supervision and Teaching, Faculty of Physical Education, \\ University of Jordan, Amman-Jordan \\ ${ }^{2}$ Department of Biological Sciences, Faculty of Science, Al al-Bayt University; AL-Mafraq-Jordan \\ 3Department of Medical chemistry\& phamacognosy, Faculty of Pharmacy, \\ Applied Sciences University, Amman-Jordan
}

\begin{abstract}
This study was carried out to assess the lipid pattern in gymnasts of the Jordan National Team. Twelve athletes represent the National gymnastic team (4 males and 8 females, mean age $18 \pm 1$ year) were included in this study. The training period for them is not less eight years during which they trained 18 hours weekly at least. A group of healthy males and females matched for age and gender was included as control group $(\mathrm{n}=20)$. No subject had evidences of cardiovascular disease, diabetes and blood pressure .The body mass index (BMI) of the females was significantly $(\mathrm{p}<0.5)$ lower than that of the control $(22.5 \pm 3.21,18.9 \pm 0.9$ respectively). It was found that the majority of the males had lower total cholesterol, triglycerides, and great decreasing in LDL cholesterol and significantly $(\mathrm{p}<0.5)$ raising in HDL cholesterol, $(164.3 \pm 19.8,85.9 \pm 34.3,49.8 \pm 6.5,80.4 \pm 30.8$ respectively) comparing to the control group (186.6 $\pm 20.7,171.6 \pm 34.3,44.5 \pm 5.7,127.2 \pm 18.2$ respectively). In females a similar fashion of lipid profile was also found with a great reduction in LDL-C level .Our result suggest that gymnastics exercise affect blood cholesterol and other lipids in a positive way, by regulating the metabolism of all lipids in the blood and increasing fat oxidation during training results in an adaptive mechanism for body weight maintenance. Also increasing HDL-C level in males and females gymnasts has protective value against cardiovascular diseases such as ischemic stroke and myocardial infarction by carrying cholesterol from the body's tissues and remove cholesterol from atheroma within arteries and transport it back to the liver for excretion or re-utilization.
\end{abstract}

Key words: Gymnasts, Lipid profile, Cholesterol, TG, HDL, LDL, BMI

\section{INTRODUCTION}

Physical exercise is the performance of some activity to develop or maintain physical fitness and overall health. It is often directed toward also honing athletic ability or skill. Frequent and regular physical exercise is an important component in the prevention of some of the diseases of affluence such as heart disease, cardiovascular disease, Type 2 diabetes, obesity and hypercholesteremia ${ }^{[1,2]}$. Serum lipid levels may be associated with age, gender, dietary habits and some primary genetic defects. Low cholesterol levels characterize some rare hereditary disorders such as abetalipoproteinemia, familial hypobetalipoproteinemia and chylomicrone retention disease. Furthermore, low plasma levels of TC, with or without hypertriglyceridemia-4 0 -have been frequently described in a variety of disorders. The main metabolic benefits of regular physical activity are a rise in high density lipoprotein cholesterol (HDL-C) levels, a lowering of triglycerides (TG) and low density lipoprotein cholesterol (LDL-C) levels, and higher insulin sensitivity to fasting glucose. In addition, exercise training increases plasma clearance of postprandial lipoproteins, such as chylomicrons ${ }^{[3]}$. Chylomicrons transport dietary lipids from the intestine into the systemic venous system. Endurance exercise also increases fat oxidation, enhances dietary TG turnover, and increases HDL-C concentrations ${ }^{[4]}$.

However, several studies found ${ }^{[5]}$ that after the acute metabolic effects of exercise, i.e. $60 \mathrm{~h}$ after the last training session, there was no further effect of training on postprandial lipidemia, insulin sensitivity, and PlLPL activity). Therefore, frequent exercise is needed to

Corresponding Author: Dr: Kamal Mansi Department of Biological Sciences, Faculty of Science, Al al-Bayt University; P.O Box Jordan-Amman 11196 P.O Box 9636, AL-Mafraq-Jordan Tel: 0096265151261 
maintain metabolic benefits of endurance training ${ }^{[6]}$. Metabolic effects of training and 4 raining have been studied in obese ${ }^{[8]}$, sedentary ${ }^{[9]}$ lean ${ }^{[9,10]}$. and aerobically fit subjects ${ }^{[11]}$. Most sports are anaerobic in nature. Only the long term endurance sports such as cycling, swimming, and running are largely aerobic ${ }^{[12]}$. Gymnastics is an acyclic sport which means that the same movements are not repeated over and over ${ }^{[13]}$. There are numerous benefits to cyclic, long-term endurance sports but variety is generally not one of them. One of the major benefits of gymnastics activity is that it subjects the gymnast's body to a wide variety of stimuli. Repeating the same movement patterns over and over has recently been questioned ${ }^{[14]}$ and the generally assumed superiority of aerobic training has been shown to be illusory for many areas of fitness, particularly with regard to weight loss ${ }^{[15]}$. The flexibility demands of gymnastics are probably the most significant and unique aspects of gymnastics that serves to separate gymnastics from most other sports ${ }^{[16]}$.In this study we examined the lipid profile of the Jordanian national team of gymnastics to reveal the effect of this sport on the lipid profile of the players. Moreover, this study aims to endeavor the gender effects on the lipid profile among gymnasts.

\section{MATERIAL AND METHODS}

Experimental subjects: Twelve gymnasts (4 males and 8 females, mean age $18 \pm 1$ year) with training experience of at least 8 years and with a minimal training activity of eighteen training hours per week were included in this study. Group of healthy male and female adolescents (control group), matched for age and gender was also included $(\mathrm{n}=20)$. No subject revealed evidences of cardiovascular disease, diabetes (fasting glucose $<7 \mathrm{mmol} /$ liter) or hypertension (blood pressure $<140 / 90 \mathrm{~mm} \mathrm{Hg}$ ) when tested by specialized physicians.

Blood collection: The blood samples were collected in plain tubes from the gymnasts and control group from the antecubital vein between 8 to 10 a.m., in a sitting position after 12 hours of fasting. Blood samples were then allowed to clot, and then serum was obtained by centrifuging at 4000rpm (Cenformix).

Methods: Serum lipid profile was examined after 12 hour of fasting, including: Total Cholesterol (TC); Triglycerides (TG); High-Density Lipoprotein Cholesterol (HDL-C) Low-Density Lipoprotein Cholesterol (LDL-C). Standard biochemical procedures were used. TC, TG, LDL-C and HDL-C of all the subjects were evaluated by using commercial analytical kits from Sigma (St. Louis, Mo, USA).

Statistical analyses: All data were expressed as means \pm SD. Statistical analyses were calculated using SPSS. ANOVA test was performed to examine the differences between males and females on one hand and between experimental groups and control groups on the other hand. This technique compares variability across males and females in the experimental group and control group.

\section{RESULTS AND DISCUSSION}

Several parameters of lipid metabolism appeared strongly dependent on the training level of athletes, namely leptin concentration ${ }^{[17]}$, fatty acid oxidation level ${ }^{[18]}$, lipoprotein lipase (LPL) activity ${ }^{[19]}$ and lipoprotein levels and contents. However, the metabolic consequences of several years of hard endurance training ( $>10$ year and $>20$ hour per week) on lipid profile during detraining, i.e. when elite athletes definitively stop their sports careers, have not been extensively investigated. Due to metabolic disorders such as insulin resistance and lipoprotein redistribution, sedentary, aerobically fit subjects who stop physical activity for several months regain the body fat mass they maintained a persistently low level during training ${ }^{[19]}$ despite a reduction of energy intake and the percentage of calories from dietary fat ${ }^{[20]}$. Our results Table 1 revealed that there is significant difference in cholesterol levels between males in the experimental group and control group $(164.2 \pm 19.75,186.6 \pm 20.7$ respectively). Moreover, significant difference $(\mathrm{p}<0.5)$ between females in the experimental group $(128.6 \pm 11.5)$ and the control group $(175.00 \pm 7.5)$ was found. In terms of the difference between males and females. Table 1 clearly shows that there is significant difference $(\mathrm{p}<0.5)$ between them $(164.3 \pm 19.8$ and $128.6 \pm 11.5$ ) respectively. However, there is a slight difference between males and females in the control group (186.6 \pm 20.7 and $175.0 \pm 7.5$ respectively). The table also indicates that there is significant difference $(p<0.5)$ in triglycerides levels between males in the experimental group and males in the control group with means $85.9 \pm 34.3$ and $171.6 \pm 34.3$ respectively. Regarding females, however, there is difference between experimental group and control group (68.8 \pm 8.3 and $79.3 \pm 4.7$ respectively). In contrast to triglycerides the results of HDL, presents in Table 1, revealed that there is slight difference between males in the experimental and control groups with means of 
Am. J. Applied Sci., 5 (6): 742-746, 2008

Table 1: Serum Lipid profile levels and BMI mean \pm SD of male and female gymnasts and control

\begin{tabular}{|c|c|c|c|c|}
\hline & \multicolumn{2}{|c|}{ Males $(n=4)$} & \multicolumn{2}{|c|}{ Females $(\mathrm{n}=12)$} \\
\hline & Experimental \pm SD & Control $\pm \mathrm{SD}$ & Experimental \pm SD & Control \pm SD \\
\hline Cholesterol mg/dl & $164.3 \pm 19.8^{*}$ & $186.6 \pm 20.7^{*}$ & $128.6 \pm 11.5^{*}$ & $175.0 \pm 7.5$ \\
\hline triglycerides mg/dl & $85.9 \pm 34.3^{*}$ & $171.6 \pm 34.3^{*}$ & $68.8 \pm 8.3 *$ & $79.3 \pm 4.7$ \\
\hline HDL-C mg/dl & $49.8 \pm 6.5^{*}$ & $44.5 \pm 5.7 *$ & $46.5 \pm 3.5^{*}$ & $34.8 \pm 6.6$ \\
\hline LDL-C mg/dl & $80.4 \pm 30.8^{*}$ & $127.2 \pm 18.2 *$ & $61.7 \pm 4.45 *$ & $78.3 \pm 4.5$ \\
\hline BMI & $24.7 \pm 0.4$ & $25.2 \pm 1.7$ & $18.9 \pm 0.9 *$ & $22.5 \pm 3.2$ \\
\hline
\end{tabular}

$49.8 \pm 6.5$ and $44.5 \pm 5.7$ respectively. In relation to females, nevertheless, there is great difference $(\mathrm{p}<0.5)$ between experimental group $(46.5 \pm 3.5)$ and control group (34.8 \pm 6.7$)$. It is worth noticing that there is no great difference between males and females in total with means $43.5 \pm 5.9$ and $37.6 \pm 37.6$ respectively. Our results indicate that there is significant difference $(\mathrm{p}<0.5)$ in LDL between males in the experimental group and the control group with means $80.4 \pm 30.8$ and 127.2 \pm 18.2 . However, there is slight difference between females in the experimental and the control group $(61.7 \pm 4.5$ and $78.3 \pm 4.5$ respectively). In terms of difference between both groups with means $103.8 \pm 34.3$ and $70.1 \pm 9.5$ respectively. Our study indicates that there is no difference in body mass index (BMI) between males in experimental group $(24.7 \pm 0.4)$ and the control group $(25.2 \pm 1.7)$ but there is slight difference between females in the experimental group and the control group $(18.9 \pm 0.9,22.5 \pm 3.2$ respectively). Numerous studies documented the relation of increased physical activity with an improved cardiovascular risk factor profile in adults. In particular these studies have shown the overall benefit of physical activity as gymnastics in reducing the risk of developing coronary heart disease, especially due to the reduction of arterial blood pressure levels [21-25]. Inflammation and coagulation markers ${ }^{[26,27]}$. In our study we observed that males showed a reduction that varied from $6.8 \%$ to $98 \%$, with the most significant reduction in triglycerides $98 \%$, on the other hand, HDL-C was significantly increased. In female, we also observed a reduction from 13.9 to $60 \%$ in cholesterol, triglycerides and LDL-C, with the most significant reduction in LDH-C 60\%. While HDL-C significantly increased. John S et al, 2003 [28]. Evaluated the effect of several types of physical activity on lipids levels. Particularly, they observed that compared to sedentary physically active men had a reduction that varied from 3 to $11 \%$, in all lipids measurements. In women they also observed a reduction starting from 5 to $18 \%$, in all lipids, with the most significant reduction in triglycerides $18 \%$. However, our results revealed that the benefits from Gymnastics on total cholesterol, triglycerides and LDL cholesterol levels could be explained by the reduction in body mass index and the decreased unbalanced food habits among gymnasts. Our findings are in accordance with several other observational studies ${ }^{[29-34]}$ that reported a no significant effect of physical activity on lipids levels, by the exception of HDL cholesterol and triglycerides. Regarding the intensity of physical activity that is needed for a considerable lowering on lipids levels several investigators report that positive changes in HDL cholesterol levels observed in those who were highly exercised ${ }^{[22.34]}$. Although, it is difficult to compare results among studies, because different methods were used to classify physical activity levels, our study revealed that even intensive physical activity levels was associated with a significant increasing in HDL cholesterol levels, among females. It is widely accepted that exercise affects blood cholesterol and other lipids in a positive way, by regulating the metabolism of all lipids in the blood. Several studies demonstrate that exercise raises HDL-cholesterol in the blood. Gymnasts showed lower body mass index, fat mass and also a hypoenergetic diet, with higher supply in protein and saturated fat ${ }^{[35]}$. We conclude that gymnastics exercises affect blood cholesterol and other lipids in a positive way, by regulating the metabolism of all lipids in the blood and increased fat oxidation during training results in an adaptive mechanism for body weight maintenance. Also increasing HDL-C level in males and females gymnasts has protective value against cardiovascular diseases such as ischemic stroke and myocardial infarction by carrying cholesterol from the body's tissues and remove cholesterol from atheroma within arteries and transport it back to the liver for excretion or re-utilization which is the main reason why HDL-bound cholesterol. Cholesterol is insoluble in blood, but is transported in the circulatory system bound to one of the varieties of lipoprotein (HDL) as spherical particles which have an exterior composed mainly of water-soluble proteins. Our future work will be concentrate on the study of the effects of some aerobic sports on lipid profile. 


\section{ACKNOWLEDGMENT}

The authors are grateful to her highness princes Rahma Bent ALHassan (President of Jordan national team) for her endless support. Also we thank all the Jordan gymnasts' national teams for their help in accomplish this work.

\section{REFERENCES}

1. Swain, D.P. and B.A. Franklin, 2006. Comparison of cardioprotective benefits of vigorous versus moderate intensity aerobic exercise. Am. J. Cardiology, 9: 141-147.

2. Murtagh, E.M., C.A.G. Boreham, A. Nevill, L.G. Hare and M.H. Murphy, 2005. The effects of 60 minutes of brisk walking per week, accumulated in two different patterns, on cardiovascular risk. Preventive Medicine, 41: 92-97.

3. Mankowitz K, R. Seip, C.F. Semenkovich, A.Daugherty and G. Schonfeld, 1992. Short-term interruption of training affects both fasting and post-prandial lipoproteins. Atherosclerosis 95: 181-189.

4. Pasman W.J, W.H. Saris, E.Muls, G. Vansant and M.S. Westerterp-Plantenga, 1999 ...Effect of exercise training on long-term weight maintenance in weight-reduced men. Metabolism 48:15-21.

5. Herd S.L., A.E. Hardman, L.H. Boobis and C.J. Cairns, 1998.The effect of 13 weeks of running training followed by $9 \mathrm{~d}$ of detraining on postprandial lipaemia. Br. J. Nutr. 80: 57-66.

6. Hardman A.E., J.E. Lawrence and S.L. Herd, 1998. Postprandial lipemia in endurance-trained people during a short interruption to training. J. Appl. Physiol 84: 1895-1901.

7. Despres J.P., Couillard C., J.Gagnon, J. Bergeron, A.S. Leon, D.C. Rao, J.S. Skinner, J.H. Wilmore and C. Bouchard, 2000. Race, visceral adipose tissue, plasma lipids, and lipoprotein lipase activity in men and women: the Health, Risk Factors, Exercise Training, and Genetics (HERITAGE) family study. Arterioscler Thromb. Vasc. Biol. 20: 1932-1938.

8. Halbert J.A., C.A. Silagy, P. Finucane, R.T. Withers, P.A. Hamdorf, 1999. Exercise training and blood lipids in hyperlipidemic and normolipidemic adults: a meta-analysis of randomized, controlled trials. Eur. J. Clin. Nutr. 53: 514-522.
9. Motoyama M., Y. Sunami, F. Kinoshita, T. Irie, J. Sasaki, K. Arakawa, A. Kiyonaga, H. Tanaka and M. Shindo, 1995. The effects of long-term low intensity aerobic training and detraining on serum lipid and lipoprotein concentrations in elderly men and women. Eur. J. Appl. Physiol. Occup. Physiol. 70: 126-131.

10 Hickner R.C., S.B. Racette, E.F. Binder, J.S. Fisher and W.M. Kohrt, 2000 Effects of 10 days of endurance exercise training on the suppression of whole body and regional lipolysis by insulin. J. Clin. Endocrinol. Metab. 85: 1498-1504.

11. Ziogas G.G., T.R. Thomas and W.S. Harris, 1997. Exercise training postprandial hypertriglyceridemia and LDL subfraction distribution. Med. Sci. Sports Exerc. 29: 986-991.

12. Ainslie P., T. Reilly and K. Westerterp, 2003. Estimating Human Energy Expenditure: A review of techniques with particular reference to doubly labelled water. Sports Med. 33: 683-698.

13. Bompa T.O., 1990. Theory and methodology of training, 2nd edn. Dubuque, IA: Kendall/Hunt,

14. Skiff, M.C., and Y.V. Verkhoshansky, Super training 1993. Johannesburg, South Africa: The School of Mechanical Engineering, University of Witwatersrand,

15. Blix, G.G, 1995. The role of exercise in weight loss. Behav. Med. 21: 31-39.

16. Sands, W.A, 1996. Physical abilities profilesNational TOPs testing. Technique 14: 15-20, 1996.

17. Haluzik M., D. Haluzikova, L. Boudova, J. Nedvidkova, M. Barackova, P. Brandejsky, V. Novotny and Z. Vilikus, 1999. The relationship of serum leptin levels and parameters of endurance training status in top sportsmen. Endocr. Res. 25: 357-369.

18. Bergman B.C, G.E. Butterfield, E.E Wolfel, G.A. Casazza, G.D. Lopaschuk, G.A. Brooks, 1999 Evaluation of exercise and training on muscle lipid metabolism. Am J Physiol 276: 106-E117.

19. Hong Y., T. Rice, J. Gagnon, M. Province, C. Bouchard, A.S. Leon, J.S. Skinner, J.H Wilmore, D.C Rao and J.P Despres, 2000. Familiarity of triglyceride and LPL response to exercise training: the HERITAGE study. Med. Sci. Sports Exerc. 32: 1438-1444.

20. Pasman W.J, M.S. Westerterp and W.H. Saris, 1999. The effect of body weight changes and endurance training on $24 \mathrm{~h}$ substrate oxidation. Int. J. Obes. Relat. Metab.Disord. 23: 1223-1232. 
21. Paffenbarger R. and I.M. Lee, 2000. Physical Activity and Coronary Heart Disease in Men.The Harvard Alumni Health Study. Circulation. 102:975

22. Lakka T.A., J.M. Venalainen, R. Rauramaa, et al., 1994. Relation of leisure-time physical activity and cardiorespiratory fitness to the risk of acute myocardial infarction. N. Engl. J. Med. 330:1549-1554.

23. Lee I.M, C.C. Hsieh, R.S. Paffenbarger, 1995. Exercise Intensity and Longevity an Men: the Harvard Alumni Health Study. JAMA. 273: 1179-1184.

24. Pitsavos C., D.B. Panagiotakos, C. Chrysohoou, P.F. Kokkinos, J. Skoumas, I. Papaioannou, C. Stefanadis, P.K. Toutouzas, 2002. The Effect of the Combination of Mediterranean Diet and Leisure time Physical Activity on the Risk of Developing Acute Coronary Syndromes, in Hypertensive Subjects. J. Hum. Hypert.. 16:517-524.

25. Pate R.R., M. Pratt, S.N.Blair et al., 1995. Physical activity and public health: a recommendation from the Centers for Disease Control and Prevention and the American College of Sports Medicine. JAMA. 273: 402-407.

26. Pitsavos C., C. Chrysohoou, D.B Panagiotakos, J. Skoumas, A. Zeimbekis, P. Kokkinos, C. Stefanadis, P.K. Toutouzas, 2003. Association of Leisure-time Physical Activity on Inflammation Markers (C-reactive protein, White-blood Cell count, serum Amyloid A and Fibrinogen), in Healthy subjects (from the ATTICA Study). Am J Cardiol. 2: 368-370.
27. Wannamethee S.G., G.D.O. Lowe, P.E. Whincup, A. Rumley, M. Walker, L. Lennon, 2002. Physical activity and hemostatic and inflammatory variables in elderly men. Circulation 105: 1785-1790.

28. John S., P. Christos, Demosthenes B Panagiotakos, C. Christina, Z. Akis, P. Ioanna, T. Marina, Pavlos and S. Christodoulos, 2003. Physical activity, high density lipoprotein cholesterol and other lipids levels, in men and women from the ATTICA study. Lipids health and Disease 108: 1416-1424.

29. Hsieh S.D., H. Yoshinaga, T. Muto and Y. Sakurai, 1998 Regular physical activity and coronary risk factors in Japanese men. Circulation.; 97:661-665

30. Bijnen F.C., E.J. Feskens, C.J. Caspersen, et al., 1996. Physical activity and cardiovascular risk factors among elderly men in Finland, Italy and the Netherlands. Am. J. Epidemiol. 143: 553-561.

32. Pols M.A., P.H. Peters, J.W. Twisk et al., 1997. Physical activity and cardiovascular risk disease risk profile in women. Am. J. Epidemiol. 146:322-328.

33. Stefanick M.L, S. Mackey, M. Sheehan, N. Ellsworth, W.L. Haskell and P.D.Wood,1998. Effects of diet and exercise in men and postmenopausal women with low levels of HDL cholesterol and high levels of LDL cholesterol. N. Engl. J. Med.; 339: 12-20.

34. Kokkinos P.F, J.C. Holland, P. Narayan, J.A. Colleran, C.O. Dotson, V. Papadimitriou, 1995. Miles run per week and high-density lipoprotein cholesterol levels in healthy, middle-aged men. A dose-response relationship. Arch. Int. Med.; 155: 415-420.

35. Abramson J.L, V. Vaccarino, 2002. Relationship between physical activity and inflammation among apparently healthy middle-aged and older US adults. Arch Intern Med. 162:1286-1292. 